\title{
Elucidating gene signatures that control the circadian rhythm in cyanobacteria using bioinformatics methods
}

Tulip Nandu, Meeta Pradhan, Mathew J Palakal

From Eighth International Society for Computational Biology (ISCB) Student Council Symposium 2012 Long Beach, CA, USA. 13-14 July 2012

\section{Background}

The circadian rhythm, or biological "clock," allows the organism to anticipate and prepare for the changes in the physical environment. Studies have found that the internal clock consists of an array of genes and the protein products they encode, which regulate various physiological processes throughout the body. Cyanothece sp. ATCC 51142 is an organism that has both photosynthetic (producing oxygen) and nitrogen fixing ability. It has developed a temporal regulation in which N2 fixation and photosynthesis occur at different times throughout a diurnal cycle with very high levels of $\mathrm{CO} 2$ fixation during the light and high levels of N2 fixation in the dark. The mechanisms underlying the circadian rhythm and the signature genes elucidating this mechanism are addressed in this research.

\section{Objective}

The objective is to integrate gene expression data with data and knowledge from prior studies using bibliomics techniques, in the de novo construction of quasi-complete regulatory networks to identify gene signatures in

Table 1 Signature genes expressed during the day.

\begin{tabular}{|c|c|}
\hline Function & Genes \\
\hline \multicolumn{2}{|l|}{ Photosynthesis Cluster } \\
\hline Photosystem II & psbD2, psbO*, psbA1, psbA3, psbF, psbE, psbY, psbA4*, psbA1*, psbA2*, petA*, \\
\hline Cytochrome Family & psbV, petB*, petJ \\
\hline Ferredoxin Type & petF1, petF5, \\
\hline Carbon Fixation & glcD, glcE, glcF, rbcL \\
\hline Thiamine Biosynthesis & thiC, thiE, thiL, thiOG \\
\hline Pantothenate Biosynthesis & panB, panD \\
\hline CoA biosynthesis & ilvB \\
\hline Fatty Acid Biosynthesis & $a c c D, f a b l, f a b G$ \\
\hline Amino acyl - tRNA biosynthesis & 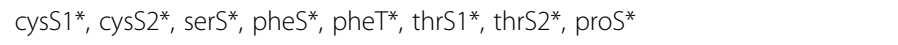 \\
\hline DNA replication & $\operatorname{lig} A^{*}, p o l A^{*}, r n h A^{*}$ \\
\hline Glyoxylate and dicarboxylate metabolism & purU, glcD, folD, glcE, glcF \\
\hline Butanoate Metabolism & pdhA, ilvN, ilvB, gabD \\
\hline
\end{tabular}

* - Genes that are differentially expressed

\footnotetext{
* Correspondence: mpalakal@iupui.edu

School of Informatics, Indiana University-Purdue University, Indianapolis,

Indiana, 46204, USA
}

(c) 2012 Nandu et al; licensee BioMed Central Ltd. This is an Open Access article distributed under the terms of the Creative Commons B. Wed Central Attribution License (http://creativecommons.org/licenses/by/2.0), which permits unrestricted use, distribution, and reproduction in any medium, provided the original work is properly cited. 
Table 2 Signature genes expressed during the night

\begin{tabular}{ll}
\hline Function & Genes \\
\hline Sulfur Metabolism & cysC \\
Amino acid Biosynthesis & cysK, ilvG \\
Galactose Metabolism & galE1, galE2 \\
Riboflavin Metabolism & ribA, ribC, ribD \\
\hline
\end{tabular}

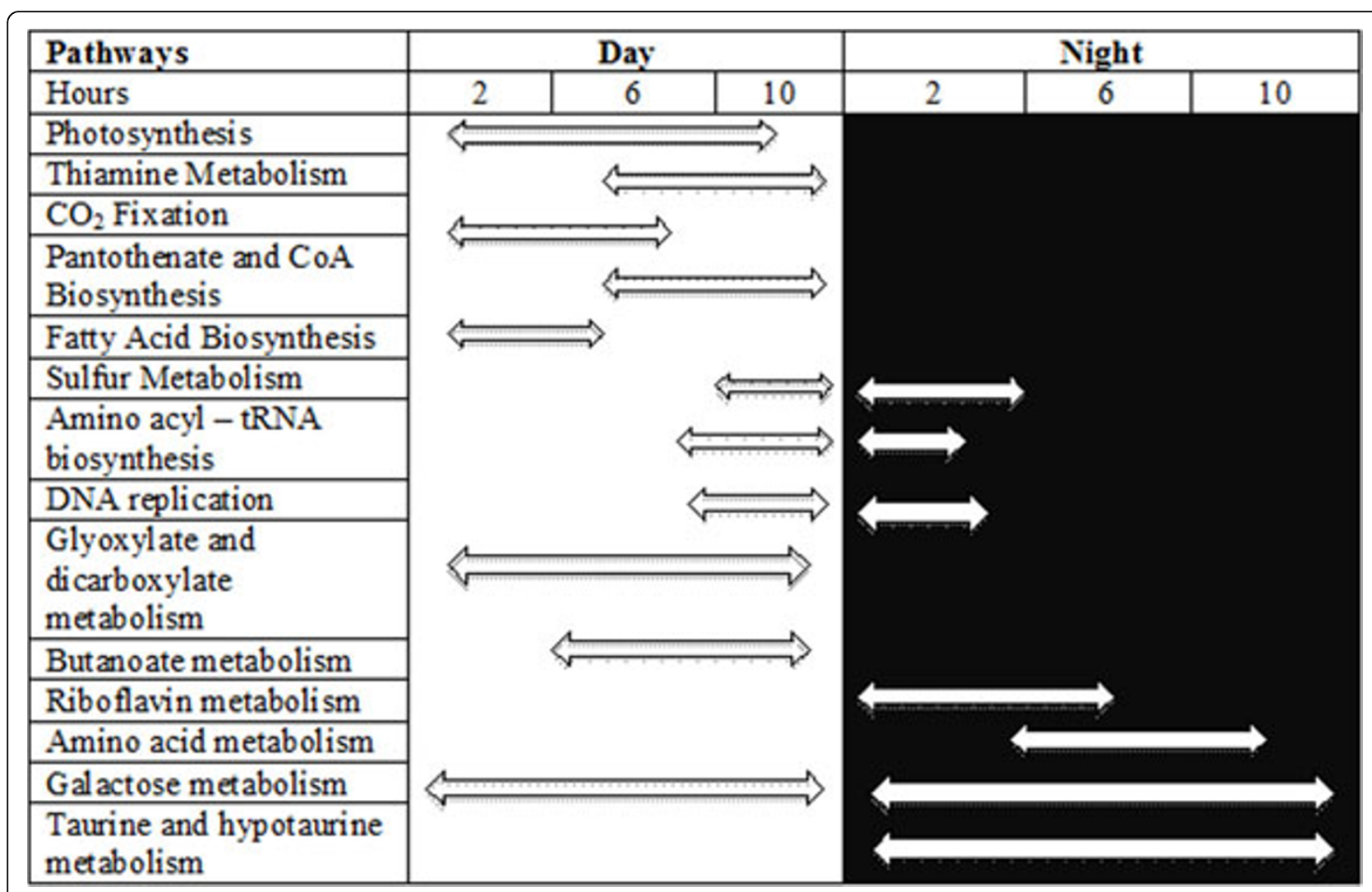

Figure 1 Expression of pathways during the time period

functional motifs and elucidate their role in circadian rhythms in Cyanothece sp. ATCC 51142.

\section{Results}

Tables 1 and 2 show the signature genes identified from topological analysis that lead to a specific pathway in Cyanothece sp. ATCC 51142. Figure 1 shows the pathways and their peak expression during the time of the day or night depending on the signature genes.

\section{Conclusions and potential implications}

The analyses show that most of the top ranked genes in the topological analysis was obtained from text mining. This shows that expression data alone is not a good measure to study the biochemical pathways and signature genes in an organism (specially less studied species).
The algorithms and methodology developed can be extrapolated to any organism, which is less studied to study their gene regulatory elements and also elucidate gene signatures that lead to specific biochemical pathways in a particular organism.

Published: 14 December 2012

doi:10.1186/1471-2105-13-S18-A9

Cite this article as: Nandu et al:: Elucidating gene signatures that

control the circadian rhythm in cyanobacteria using bioinformatics methods. BMC Bioinformatics 2012 13(Suppl 18):A9. 